\begin{tabular}{|c|c|c|c|}
\hline \multirow{2}{*}{$\begin{array}{r}\text { Case Reports in } \\
\text { Gastroenterology }\end{array}$} & \multicolumn{2}{|c|}{ Case Rep Gastroenterol 2017;11:109-113 } & \multirow[b]{2}{*}{$\begin{array}{l}\text { Karger } \\
\text { Open access }\end{array}$} \\
\hline & $\begin{array}{l}\text { DOI: } 10.1159 / 000457789 \\
\text { Published onlIne: Vlarch } 3,2017\end{array}$ & $\begin{array}{l}\text { (C) } 2017 \text { The Author(s)Published by S. } \\
\text { Karger AG, Basel } \\
\text { www.karger.com/crg }\end{array}$ & \\
\hline & $\begin{array}{l}\text { This article is licensed under } \\
\text { International License (CC BY-N } \\
\text { Usage and distribution for comm }\end{array}$ & $\begin{array}{l}\text { nons Attribution-NonCommercial } 4 . \\
\text { ger.com/Services/OpenAccessLicense) } \\
\text { uires written permission. }\end{array}$ & \\
\hline
\end{tabular}

\title{
Perianal Pagetoid Intraepithelial Carcinoma
}

\author{
Suanga Sisodia ${ }^{a}$ Robin Boushey ${ }^{b}$ Goo Lee $^{c}$ Celia Marginean ${ }^{c}$ \\ Marcio M. Gomes ${ }^{c}$ Gaurav Bhattacharya ${ }^{a}$ Kristopher Dennis $^{a}$ \\ ${ }^{a}$ Division of Radiation Oncology, The Ottawa Hospital and the University of Ottawa, \\ Ottawa, ON, Canada; ${ }^{b}$ Division of General Surgery, The Ottawa Hospital and the University \\ of Ottawa, Ottawa, ON, Canada; ' ${ }^{\mathrm{C}}$ epepartment of Pathology and Laboratory Medicine, The \\ Ottawa Hospital and the University of Ottawa, Ottawa, ON, Canada
}

\section{Keywords}

Colorectal carcinoma - Extramammary Paget disease - Intraepithelial carcinoma - Paget cells . Paget disease $\cdot$ Pagetoid intraepithelial carcinoma - Perianal region

\begin{abstract}
Extramammary Paget disease (EMPD) involving the perianal region is rare and challenging to manage. Primary EMPD involves stand-alone noninvasive lesions within the epidermis, while secondary EMPD involves phenotypically similar lesions derived from separate underlying malignancies. Differentiating between primary and secondary EMPD is challenging when no underlying malignancies are detected during workup. Continued reporting of perianal EMPD cases is encouraged so that risk stratification can be improved and patients can be managed with an appropriate level of aggressiveness. Herein, we report the case of a 74-year-old woman who chose aggressive surgical management after being diagnosed with perianal pagetoid intraepithelial carcinoma from a suspected occult underlying primary colorectal tumor.

(C) 2017 The Author(s)

Published by S. Karger AG, Basel
\end{abstract}




\section{Introduction}

Extramammary Paget disease (EMPD) is a rare intraepithelial carcinoma most commonly seen in the elderly, notably postmenopausal white women [1, 2]. EMPD lesions are often in areas with apocrine glands, such as the anogenital region [1, 2], with anorectal lesions suggested to be the most aggressive form [1]. Lesions typically present as scaly eczema-like rashes, sometimes de-pigmented and filled with fluid [1-6].

Primary EMPD involves stand-alone noninvasive lesions within the epidermis, compared with secondary EMPD which involves phenotypically similar lesions derived from separate underlying primary malignancies [2]. Secondary EMPD lesions have been suggested to account for $29 \%$ of all EMPD cases, with the most common underlying primary malignancies being of colorectal and genitourinary origin $[1,2]$. Reports have suggested that EMPD involving the perianal skin is more likely to be associated with an underlying primary malignancy than EMPD involving other sites [3]. However, due to the rarity of these lesions, especially those in the perianal area, their natural history is incompletely understood [4].

A challenging aspect of managing secondary EMPD involving the perianal skin is that an underlying primary malignancy may escape detection during workup, and not be identified until years following initial management [3]. Ideally, EMPD lesions would be classified on initial presentation as being at either a high or low risk of having an associated primary underlying malignancy. Unfortunately, risk stratification based on features of the EMPD lesions themselves is suboptimal. This complicates treatment decision-making, when no underlying malignancies are detected clinically.

Herein, we report a rare case of perianal pagetoid intraepithelial carcinoma from a suspected occult underlying colorectal primary tumor to demonstrate the challenges posed by such lesions with respect to workup, diagnosis, and treatment decision-making.

\section{Case Summary}

A 74-year-old woman presented with persistent constipation, bloating, and a 3-week history of a nonhealing perianal lesion she presumed was an abrasion. She had a history of emphysema, diverticular disease, gastric ulcer, cataract repair, cesarean section, and a remote right breast pT1N0 ER/PR-positive HER-2/neu-negative lobular carcinoma treated successfully with mastectomy and adjuvant hormonal therapy. She had a family history of lung and liver cancer, but not of colorectal cancer.

On examination, there was a $4 \times 5$-cm erythematous and scaly lesion raised $5 \mathrm{~mm}$ from the normal contour of the skin, extending from the left perianal region to the anal verge. Colonoscopy, CT colonography, and pelvic MRI showed no abnormalities beyond the visible lesion. A biopsy revealed involvement of the epithelium but not the dermis, with Paget cells that were positive for CK20, CDX2, pCEA, and MUC2 and negative for CK7, MUC1, MUC5, ER, BRST2, GATA-3, S100, and Melan-A. Eight out of 8 subsequent mapping biopsies of the anal margin and verge showed similar involvement of intraepithelial carcinoma with pagetoid extension. Numerous signet ring cells infiltrated the squamous mucosa. Occasional acellular mucin pools were seen. No invasive adenocarcinoma was identified. Signet ring cells were positive for CK20, CDX2, and p53 and negative for CK7, GCDFP-15, S100, and MUC5AC. Four out of 4 mapping biopsies from the anal canal proper were negative. Overall, the clinical and pathological findings were suspicious for pagetoid infiltration of the perianal skin from a synchronous/metachronous occult colorectal primary tumor, as the immunophenotype of 
the signet ring cells is more commonly seen in secondary EMPD associated with rectal adenocarcinoma $[3,4]$.

The patient considered options including surveillance, wide local excision, and primary radiation therapy, but ultimately chose an aggressive approach and underwent an abdominoperineal resection with posterior vaginectomy, bilateral salpingo-oophorectomy, and vertical rectum abdominis muscular cutaneous flap reconstruction of the pelvic inlet and perineal zones with neoperineal body creation. Pathological examination confirmed a $7-\mathrm{cm}$ focus of Paget cells immunophenotypically consistent with secondary disease that involved the perianal skin, anorectal junction, and vagina. No associated primary malignancy was identified within the anus, rectosigmoid colon, vagina, ovaries, or fallopian tubes. Paget cells were positive for CK20, CDX2, MUC2, pCEA, and EMA and negative for CK7, GATA, MUC5AC, GCDFP-15, p40, S100, and HMB45. Rare Paget cells with extravasated mucin were suspicious for involving the superficial dermis, but otherwise there was no definitive invasion into the dermis or submucosa (Fig. 1, Fig. 2). Twenty-nine regional lymph nodes were examined and were negative for malignancy. No lymphovascular or perineural invasion were identified.

The patient is presently well and at the time of submission had no evidence of recurrence or underlying malignancy, 6 months following surgery. A careful surveillance program is planned.

\section{Discussion}

EMPD affecting the perianal region is relatively rare. Patients can complain of itchiness, pain, and bleeding in affected areas. EMPD lesions have been reported to be associated with underlying malignancies in up to $29 \%$ of cases, with perianal lesions most commonly being associated with underlying gastrointestinal tumors [1]. Thorough workup, including endoscopic assessment and imaging to rule out asymptomatic underlying primary malignancies, is recommended.

For patients without obvious underlying malignancies identified during workup, definitive management is influenced by the likelihood of local or distant recurrence. A depth of invasion of more than $1 \mathrm{~mm}$, invasion to the dermis, tumor thickness of $>3 \mathrm{~mm}$, extracutaneous involvement, Ki-67 and cyclin D1 expression, MUC5AC expression, anogenital location, endodermal differentiation with gastrointestinal-type glands containing dirty necrosis, mucin pools, numerous signet ring cells, CK20 positivity, GCDFP-15 negativity, and loss of Ecadherin have been linked to higher rates of local recurrence and underlying malignancy in different series [1-4]. Our patient's profile contained many of these factors, and due to the extensive involvement of the mapping biopsies and fear of subsequent locoregional recurrence, she opted for aggressive surgical treatment.

Noninvasive perianal EMPD can be managed with local excision, radiation therapy, photodynamic therapy, cryotherapy, topical 5-fluorouracil ointment and imiquimod cream, while invasive disease is more often treated with aggressive surgery including abdominoperineal resection or alternatively radiation therapy with or without concurrent chemotherapy [4-6].

Lian et al. [4] reported 8 cases of perianal Paget disease with underlying anorectal malignancies. All 8 underwent abdominoperineal resection; 6 up front and 2 after recurrence following local excision. Goldblum et al. [3] reported 11 cases of perianal Paget disease; 5 of 11 were associated with underlying rectal tumors. One of those 5 did not have the suspected underlying primary tumor present until 30 months after local excision of the perianal dis- 
Sisodia et al.: Perianal Pagetoid Intraepithelial Carcinoma

ease. They suggested that 2 types of perianal Paget disease exist: type 1, an aggressive type with endodermal differentiation that usually shows gastrointestinal-type glands containing intraluminal dirty necrosis, numerous signet ring cells, CK20 positivity and GCDFP-15 negativity, and is more likely to be associated with synchronous or metachronous rectal adenocarcinoma; and type 2, a less aggressive primary cutaneous intraepithelial type in which Paget cells have sweat gland differentiation, lack gastrointestinal-type glands as well as intraluminal dirty necrosis, and are negative for CK20 and positive for GCDFP-15.

Continued reporting of cases of perianal EMPD is important so that risk stratification can be improved. Ideally, patients at high risk of underlying malignancy should be managed more aggressively and avoid recurrences following (for example) local excisions, and patients at lower risk should avoid unnecessarily aggressive treatments.

\section{Acknowledgements}

The authors are grateful for the support of The Arseneau-Henry Families Research Fund and the Joseph Doyle Research Fund.

\section{Statement of Ethics}

The authors have no ethical conflicts to disclose.

\section{Disclosure Statement}

The authors have no conflicts of interest to disclose.

\section{References}

1 Cohen J, Granter S, Werchniak A: Risk stratification in extramammary Paget disease. Clin Exp Dermatol 2015;40:473-478.

2 Ito T, Kaku Y, Nagae K, Nakoano-Nakamura M, Nakahara T, Oda Y, Hagihara A, Furue M, Uchi H: Tumor thickness as prognostic factor in extramammary Paget's disease. J Dermatol 2015;42:269-257.

3 Goldblum JR, Hart WR: Perianal Paget's disease: a histologic and immunohistochemical study of 11 cases with and without associated rectal adenocarcinoma. Am J Surg Pathol 1998;22:170-179.

4 Lian P, Gu W, Zhang Z, Cai G, Wang M, Xu Y, Sheng W, Cai S: Retrospective analysis of perianal Paget's disease with underlying anorectal carcinoma. World J Gastroenterol 2010;23:2943-2948.

-5 Mann J, Lavaf A, Tejwani A, Ross P, Ashamalla H: Perianal Paget disease treated definitively with radiotherapy. Curr Oncol 2012;19:496-500.

-6 Hallak M, Zouain N: Extramammary perianal Paget's disease. Case Rep Gastroenterol 2009;3:332-337. 


\section{Case Reports in Gastroenterology \\ Case Rep Gastroenterol 2017;11:109-113}
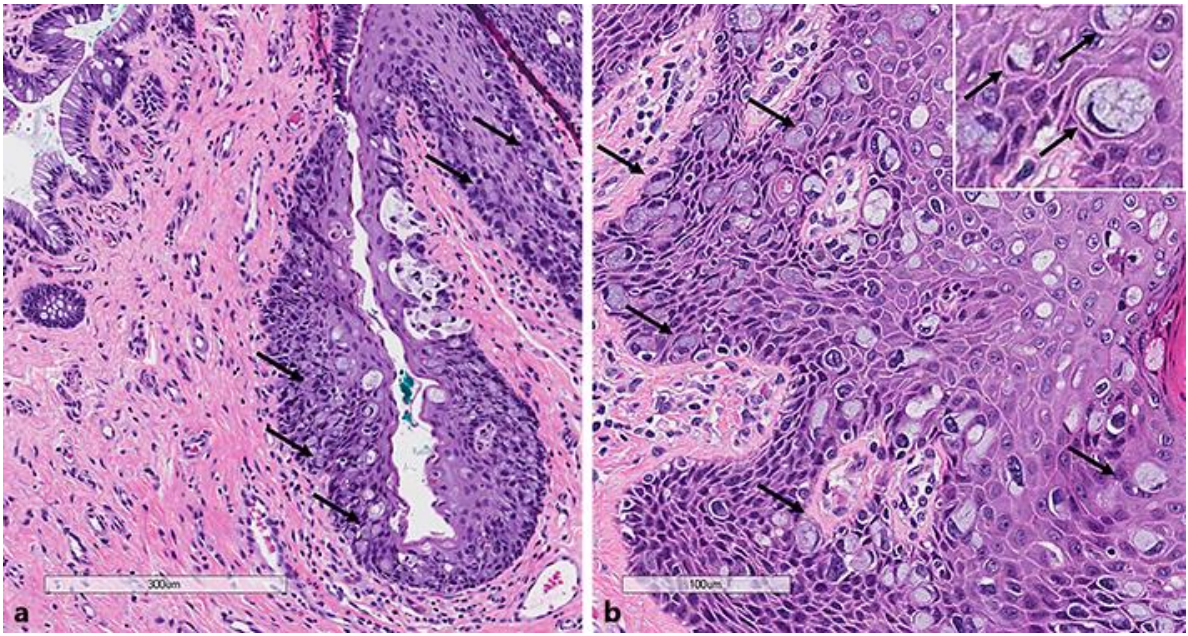

Fig. 1. Hematoxylin and eosin stains show intraepithelial spread of Paget cells involving the anal transitional zone. a The squamo-columnar junction is seen. $\mathbf{b}$ At the upper right corner (inset, $\times 40$ ) Paget cells with large cytoplasmic mucin globules and peripherally displaced nuclei are shown.

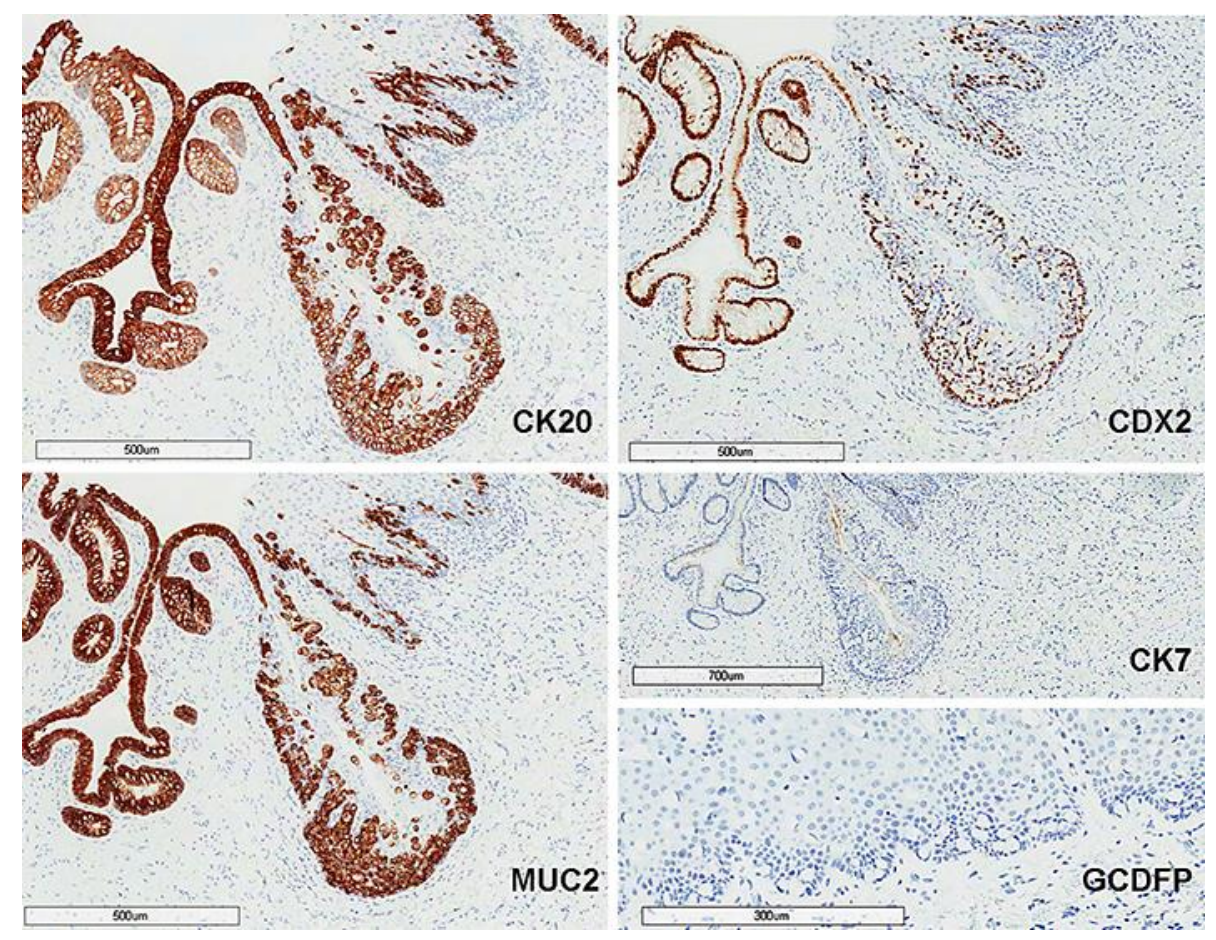

Fig. 2. Immunohistochemical stain results. The Paget cells are positive for CK20 and CDX2. The large cytoplasmic globules are positive for MUC2. The Paget cells are negative for CK7 and GCDFP-15. 